\title{
Déficits na Memória de Trabalho em Idosos com Depressão Maior: Uma Revisão Sistemática
}

\author{
Tatiana De Nardi ${ }^{1}$ \\ Breno Sanvicente-Vieira \\ Rodrigo Grassi-Oliveira \\ Pontificia Universidade Católica do Rio Grande do Sul
}

\begin{abstract}
RESUMO - A depressão em idosos é associada com prejuízos cognitivos, entretanto a extensão destes à Memória de Trabalho (MT) ainda não é consensual. Portanto, o objetivo deste estudo é revisar sistematicamente as associações encontradas entre MT e depressão em idosos. Para tanto conduzimos uma revisão sistemática dos artigos publicados entre 2000 e 2011 nas principais bases de dados internacionais. Posteriormente a aplicação dos critérios de exclusão, 17 artigos foram revisados integralmente. Os resultados apresentam evidências da associação entre depressão geriátrica e prejuízos da MT, que em alguns trabalhos ainda foram mantidos mesmo após a remissão da sintomatologia de humor.
\end{abstract}

Palavras-chave: memória operacional, memória de curta duração, depressão geriátrica, cognição, idosos, neuropsicologia

\section{Impairment of Working Memory during a Depression of the Elderly: A Systematic Review}

\begin{abstract}
Geriatric depression is related with cognitive impairments, but how this is connected to specific Working Memory deficits is still unknown. Hence, the aim of this study is to systematically review the literature about the associations between Working Memory impairments and major depression in the elderly. Thus we performed a systematic review, considering published articles in major international databases between 2000 and 2011. After exclusion criteria, 17 articles were fully reviewed. There is evidence that there is indeed an association between depression in elderly and Working Memory impairments. In addition, some articles found that such deficits are sustained even after mood symptoms remission.
\end{abstract}

Keywords: working memory, short-term memory, geriatric depression, cognition, elderly, neuropsychology

A Memória de Trabalho (MT) é definida como um sistema que permite o armazenamento temporário e a manipulação de informações necessárias para realização de tarefas complexas como a compreensão, a aprendizagem e o raciocínio (Baddeley, 2000). Ela é dita como online e com uma durabilidade de dois a três minutos. A complexidade dessa função engloba a ativação especialmente de estruturas do córtex pré-frontal dorsolateral, do córtex parietal e suas conexões: via córtex entorrinal, como o hipocampo, a amígdala e o córtex temporal inferior (Owen, McMillan, Laird, \& Bullmore, 2005).

No ciclo desenvolvimental, a MT apresenta um declínio esperado no envelhecimento. Experimentos neuropsicológicos, ao avaliar a MT em idosos saudáveis, demonstram que tais déficits são percebidos quando se utiliza tarefas de repetição de dígitos (span) em ordem inversa (Goffaux, Phillips, Sinai, \& Pushkar, 2008; Luo \& Craik, 2008), ilustrando dificuldades em atividades com aspectos mnemônicos e executivos.

Estudos que utilizam ressonância magnética funcional demonstraram que existem diferenças no desempenho da MT de idosos quando comparados com adultos jovens. Estas são especialmente relacionadas a alterações em ligações entre regiões estriatais e o córtex pré-frontal, as quais modificam

1 Endereço para correspondência: Avenida Ipiranga, 6681 prédio 11 sala 936, Partenon. Porto Alegre, RS CEP: 90619-900.

E-mail: tatydenardi@yahoo.com.br a magnitude de ativação (Nyberg, Dahlin, Stigsdotter Neely, \& Bäckman, 2009).

Pesquisas sobre a depressão em idosos identificaram prejuízos significativos da MT em relação a idosos saudáveis (Nebes et al., 2000). Entretanto, ainda existem estudos que refutam essa hipótese (Fischer et al., 2008). Algumas investigações que utilizam neuroimagem estrutural em pacientes idosos deprimidos também identificaram alterações anatômicas no córtex orbital bilateral e na substância branca do córtex pré-frontal, áreas diretamente relacionadas com o funcionamento da MT (Rozenthal, Laks, \& Egekhardt, 2004).

Sabe-se que as dificuldades na MT limitam bastante a capacidade funcional do indivíduo, prejudicando de forma importante a aprendizagem, raciocínio e decisões imediatas, portanto restringindo sua autonomia (Owen et al., 2005). A depressão, por sua vez, é uma psicopatologia que afeta a iniciativa do paciente, deixando-o menos interessado no ambiente (Mondal, Sharma, Das, Goswami, \& Gandhi, 2007) e, portanto comprometendo a motivação para se envolver em atividades cognitivamente mais complexas (Fiske, Wetherell, \& Gatz, 2009). Sendo assim, a interação desses dois fatores pode gerar grande impacto na vida do indivíduo, o que torna o tema de grande relevância, ainda mais por se observar que idosos deprimidos respondem menos ao tratamento antidepressivo, mantendo especialmente os déficits cognitivos (Fiske et al., 2009). 
A literatura vem trazendo ainda uma discussão em torno das diferenças no padrão neuropsicológico relacionado ao período de ocorrência do primeiro episódio depressivo. A depressão cujo início ocorreu já na velhice é denominada Depressão de Início Tardio e está mais associada a declínios cognitivos abruptos e severas alterações na estrutura cerebral frontoestriatal (Herrmann, Goodwin, \& Ebmeier, 2007). Em particular, estudos vêm associando a depressão de início tardio à disfunção executiva, relacionada às alterações frontais, enquanto a depressão precoce, ou que teve seu primeiro episódio antes dos 60 anos, está mais relacionada à redução do volume hipocampal (O’Brien, Lloyd, McKeith, Gholkar, \& Ferrier, 2004) e a prejuízos na memória episódica (Herrmann et al., 2007).

Apesar de existir certa concordância que a depressão em idosos provoca prejuízos na MT em relação aos idosos saudáveis, ainda existem estudos que não corroboram essa hipótese. Dessa forma, uma revisão sistemática que identifique e analise metodologicamente todos os estudos dos últimos 10 anos que investigaram a MT de idosos deprimidos torna-se uma interessante estratégia para a compreensão dos fatores que mantêm essa divergência. Portanto, este artigo objetiva responder à seguinte questão: A literatura efetivamente evidencia déficit na MT em idosos com depressão maior?

\section{Método}

Neste estudo foi realizada uma revisão sistemática de artigos empíricos nas bases de dados: MEDLINE, PsycINFO, Web of Science (ISI database), SciELO Brasil, Cochrane e LILACS, publicados nos últimos 12 anos (entre 2000 e 2011) a partir da chave de descritores: ["working memory" AND "depression" AND "elderly" OR "geriatric" OR "aging" OR “ageing” OR “aged"]. No total foram encontradas 800 referências, cujos resumos foram analisados independentemente por três avaliadores. Foram incluídos apenas artigos

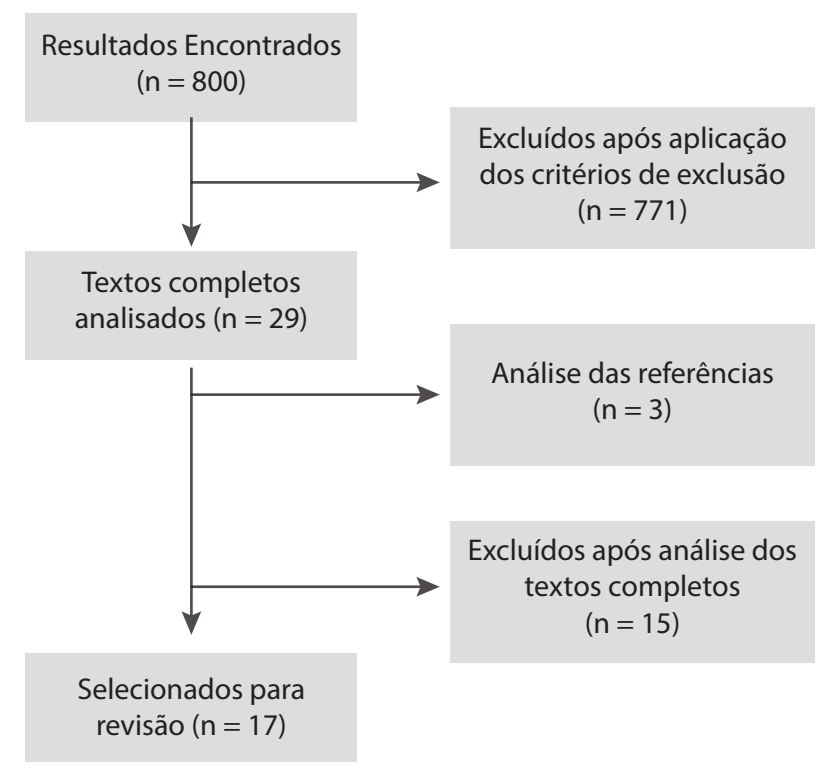

Figura 1. Diagrama de sistematização da revisão. empíricos, sejam estudos transversais ou longitudinais. $\mathrm{Na}$ primeira etapa, foram excluídos: I) dissertações, livros, ensaios teóricos e artigos de revisão; II) artigos selecionados pelo sistema de busca da base que não abordavam o tema; III) artigos escritos em outras línguas que não português, espanhol ou inglês e IV) artigos incluídos na busca, mas que não tinham em sua amostra idosos com depressão avaliados em ao menos uma tarefa de MT. Nesse momento também, foram eliminados os artigos repetidos nas diferentes bases de dados. A partir dessa seleção inicial, mantiveram-se 29 artigos no estudo, os quais foram lidos em seu texto completo. Na segunda fase da revisão excluíram-se os artigos que não haviam operacionalizado a investigação da MT através de instrumentos descritos na literatura como medidas aceitas desse constructo. Ao final dessa segunda etapa, mantiveram-se apenas 14 artigos e buscaram-se possíveis artigos nas referências bibliográficas desses. Nessa etapa encontrou-se mais três estudos. Ao fim dos procedimentos de revisão, seguindo os critérios de inclusão e exclusão, 17 artigos foram incluídos nessa revisão sistemática. A Figura 1 apresenta um diagrama da sistematização desta revisão.

\section{Resultados e Discussão}

Nesta revisão foram encontrados 17 artigos que explicitamente investigaram a MT de idosos com depressão maior conforme o diagrama da Figura 1. Dentre os selecionados, 12 evidenciam que a MT de idosos deprimidos apresenta prejuízos em relação a idosos sem psicopatologia. Nesse sentido, respondendo à pergunta norteadora deste estudo: em torno de $70 \%$ dos resultados encontrados nos artigos selecionados sugerem que a depressão em idosos impacta a MT. Uma das explicações seria que os prejuízos na MT de idosos deprimidos, seja ela avaliada através de conteúdo verbal ou visual, estariam relacionados à diminuição da velocidade do processamento da informação (Nebes et al., 2000), todavia a maioria dos estudos selecionados não testa essa hipótese explicitamente.

Salienta-se que os quatro estudos que investigaram a MT em idosos após a remissão dos sintomas depressivos concordaram que, mesmo após a remissão dos sintomas depressivos, não houve melhora no desempenho nas tarefas de MT (Delaloye et al., 2010; Nebes et al., 2001; Nebes et al., 2000; Nebes et al., 2003). Apenas um estudo preocupou-se em investigar o efeito de fármacos antidepressivos no desempenho cognitivo dos participantes (Nebes et al., 2003). Todavia os dados dessa pesquisa evidenciaram que nem a nortriptilina nem a paroxetina tiveram efeitos no desempenho cognitivo de idosos. Além disso, o desempenho nas tarefas de MT não foi influenciado nem pelo período de início da doença (tardio ou não) e nem mesmo pelo funcionamento cognitivo prévio do paciente (Nebes et al., 2003). Isso significa que, após o início da doença, de fato haveria um prejuízo na MT e mesmo quando tratados com antidepressivos ou em remissão dos sintomas de humor, os déficits na MT persistiriam. Contudo, existem alguns autores que não compactuam com essa perspectiva; por exemplo, O’Brien et al. (2004) sugere que os prejuízos da MT observados na depressão em idosos poderiam ser 
revertidos em aproximadamente seis meses. Apesar de mais otimista, essa perspectiva não encontra muitas evidências na literatura investigada nesse estudo. Sair, Welsh-Bohmer, Wagner e Steffens (2003) referem que evidências de atrofias cerebrais e doenças cerebrovasculares isquêmicas são frequentemente observadas em estudos com neuroimagem estrutural em idosos deprimidos, o que poderia justificar as perdas cognitivas não reversíveis citadas.

Dos quatro estudos analisados que utilizaram neuroimagem (Delaloye et al., 2010; O'Brien et al., 2004; Simpson, Baldwin, Burns, \& Jackson, 2001; Steffens et al., 2004) três concordaram que a MT estaria impactada pela depressão e encontraram alterações neuroestruturais em idosos deprimidos, o que poderia ser a justificativa para o prejuízo (O’Brien et al., 2004; Simpson et al., 2001; Steffens et al, 2004). Um deles (Simpson et al., 2001) ainda identificou redução do volume ventricular em idosos com depressão de início tardio. Simpson et al. (2001) encontraram grande associação dessa psicopatologia com as condições gerais de saúde do paciente, constatando que a pior saúde física estaria associada a uma redução generalizada do volume cerebral e a um prejuízo mnemônico. Especialmente, esse estudo destacou que prejuízos na MT e demais funções superiores são relacionadas à redução do volume do lobo frontal e parietal em idosos com depressão de início tardio.

Já os outros dois estudos observaram uma redução de estruturas hipocampais em idosos deprimidos (O'Brien et al, 2004; Steffens et al, 2004). O'Brien et al. (2004), por sua vez, limitam a redução referida ao hipocampo direito, pois não encontraram reduções no hemisfério esquerdo. Eles ainda discordam de Simpson e et al. (2001), mencionado que de forma geral não há diferenças volumétricas encefálicas entre os grupos de participantes deprimidos e saudáveis. É importante relatar, que em seu estudo, esses autores não se restringiram à depressão de início tardio, tendo uma amostra mista, o que poderia ser um dos fatores causadores de divergências. Eles relacionaram seus achados com um declínio na capacidade de memória episódica (O’Brien et al., 2004). Considerando a teoria de Baddeley (2000), poderia se pensar que essa alteração estrutural impactaria o funcionamento do Buffer Episódico de idosos deprimidos, uma vez que o armazenamento na memória de longo prazo episódica dependeria desse componente.

No entanto, Delaloye et al. (2010) não encontraram diferenças significativas nos resultados dos participantes em tarefas de MT. Eles avaliaram idosos com depressão de início tardio, idosos eutímicos com histórico de depressão maior recorrente; e idosos saudáveis quanto ao desempenho cognitivo e quanto à existência de diferenças de volume encefálico. Seus achados indicaram alterações estruturais apenas em idosos com depressão de início tardio, evidenciadas pela redução do volume hipocampal, do córtex cingulado anterior e por hiperintensidades periventriculares.

Dos 17 artigos incluídos, em cinco os autores não encontraram prejuízos no desempenho do grupo de idosos deprimidos em relação a um grupo controle nas tarefas de MT (Birch \& Davidson, 2007; Delaloye et al., 2010; Fischer et al., 2008; Lonie et al., 2009; von Gunten, Giannakopoulos, $\&$ Duc, 2005). Todavia nenhum desses estudos investigou mais que 30 participantes por grupo, podendo o tamanho amostral e o método de análise escolhido ter contribuído para erro tipo II. Ademais, apesar de estudos confirmarem o impacto da depressão na MT de idosos, um deles menciona que apenas a presença da psicopatologia já afetaria o desempenho cognitivo, mas não a sua gravidade (Nebes et al., 2001). Essa divergência da hipótese mais confirmada na literatura pode estar relacionada ao fato da maior parte dos participantes investigados estarem em um nível de depressão leve, podendo o resultado alterar-se na medida em que aumenta a gravidade dessa psicopatologia (Fischer et al., 2008; Lonie et al., 2009).

Alguns dos estudos incluídos compararam o desempenho de idosos deprimidos e idosos com diagnóstico de doença de Alzheimer (DA) ou outras demências. Surpreendentemente, dentre os quatro estudos que compararam grupo de idosos deprimidos, idosos saudáveis e idosos com DA ou outra demência, três não encontraram prejuízos em idosos deprimidos (Lonie et al., 2009; Murray, 2002; von Gunten et al., 2005). Nesses casos os idosos saudáveis e com depressão não apresentaram desempenho significativamente distinto. Já no grupo de idosos demenciados, isso ficou evidente, sugerindo que a depressão não seria fator determinante na perda cognitiva. Von Gunten (2005), ao comparar idosos deprimidos com demência inicial e idosos deprimidos mas sem demência, observaram que os demenciados, mesmo que em estágio inicial, apresentaram desempenho significativamente inferior aos não demenciados, os quais desempenharam-se dentro do esperado para a faixa etária.

Entretanto, o estudo de Foldi, Brickman, Schaefer e Knutelska (2003), que comparou idosos com DA, idosos deprimidos e idosos saudáveis, observou, na tarefa de memória imediata do California Verbal Learning Test (cinco ensaios de lista de palavras), que os idosos deprimidos evocaram um número significativamente menor de palavras que os saudáveis. Além disso, os deprimidos apresentaram um maior efeito de primazia, ou seja, evocaram em maior quantidade as primeiras palavras da lista. Já os idosos com DA mostraram um efeito de recência, recordando com maior facilidade os últimos números da lista. Segundo os autores, o efeito de primazia pode significar uma preservação benéfica da MT dos idosos deprimidos em relação aos com DA. Eles sugerem que uma das explicações seria a sustentada por Baddeley (2000), na qual o ensaio articulatório realizado pela alça fonológica consegue realizar sua atividade com sucesso, mantendo ativas e conseguindo transferir para memória de longo prazo as informações primeiramente apresentadas. Contudo, para Foldi et al. (2003), a grande perda dos idosos com depressão seria no desempenho significativamente inferior aos saudáveis na evocação das palavras do meio da lista. Eles mencionam que o desempenho dos idosos deprimidos se apresenta na forma de um U, com diferenças significativas entre as posições das palavras e grande prejuízo na região do meio. Aqui os autores se utilizam dos achados de Nebes et al. $(2000,2001)$ para hipotetizar que, uma vez que a velocidade do processamento é mais lenta devido à depressão, enquanto estão sendo apresentadas as palavras do meio da lista, o avaliando ainda está envolvido com o ensaio e armazenamento das primeiras palavras, tendo, por isso, um prejuízo atencional nas palavras mediais. Já as últimas, por serem recentes, ainda estão disponíveis na alça fonológica da memória de trabalho, faci- 
Tabela 1. Descrição de características e principais resultados dos estudos revisados.

\begin{tabular}{|c|c|c|c|c|}
\hline $\begin{array}{l}\text { AUTOR / } \\
\text { ANO }\end{array}$ & $\begin{array}{l}\text { número de } \\
\text { participantes }\end{array}$ & Avaliação Clínica & Avaliação da MT & $\begin{array}{l}\text { Evidência de } \\
\text { prejuízo na MT } \\
\text { de idosos } \\
\text { deprimidos? }\end{array}$ \\
\hline $\begin{array}{l}\text { Nebes et al, } \\
2000\end{array}$ & $\begin{array}{l}\text { GD: } n=19 \\
\text { GDR: } n=20\end{array}$ & $\begin{array}{l}\text { 1. Ham-D } \\
\text { 2. SCID }\end{array}$ & $\begin{array}{l}\text { 1. N- back } \\
\text { 2. CVLT } \\
\text { 3. Rey- Osterreith Complex Figure } \\
\text { immediate } \\
\text { 4. WMS III- Immediate Logical Memory }\end{array}$ & $\begin{array}{l}\text { SIM } \\
\text { GC }>\text { GD }>\text { LOGD }\end{array}$ \\
\hline $\begin{array}{l}\text { Nebes et al, } \\
2001\end{array}$ & $\begin{array}{l}\text { GD: } n=36 \\
\text { GDR: } n=17\end{array}$ & $\begin{array}{l}\text { 1.Ham- D } \\
\text { 2. DRS } \\
\text { 3. SCID }\end{array}$ & $\begin{array}{l}\text { 1. Backward Digit } \\
\text { 2. Visualspacial Tracking task } \\
\text { 3. Condition of Dual-task }\end{array}$ & $\begin{array}{l}\mathrm{SIM} \\
\mathrm{GC}>\mathrm{GD}\end{array}$ \\
\hline $\begin{array}{l}\text { Murray, } \\
2002\end{array}$ & GDP: $n=16$ & $\begin{array}{l}\text { 1. Clinical Interview based on } \\
\text { DSM IV } \\
\text { 2. ABCD } \\
\text { 3. DRS } \\
\text { 4. HAM-D } \\
\text { 5. GDS }\end{array}$ & $\begin{array}{l}\text { 1. Digit Span } \\
\text { 2. Working Memory Test Protocol (WM) }\end{array}$ & $\begin{array}{l}\mathrm{NÃO} \\
\mathrm{GC}=\mathrm{GD} \\
\mathrm{GD}>\mathrm{ADG}\end{array}$ \\
\hline $\begin{array}{l}\text { Foldi et al, } \\
2003\end{array}$ & GD: $n=20$ & $\begin{array}{l}\text { 1. CCI } \\
\text { 2.DRS } \\
\text { 3.MMSE }\end{array}$ & $\begin{array}{l}\text { 1. Californa Verbal Learning Test } \\
\text { 2. WAIS III forward and backward Digit } \\
\text { span }\end{array}$ & $\begin{array}{l}\mathrm{SIM} \\
\mathrm{GC}>\mathrm{GD}>\mathrm{GDA}\end{array}$ \\
\hline $\begin{array}{l}\text { Steffens et } \\
\text { al, } 2004\end{array}$ & GD: $n=141$ & $\begin{array}{l}\text { 1.MMSE } \\
\text { 2.DDES } \\
\text { 3.MADRS } \\
\text { 4 CGIS } \\
\text { 5. HDRS } \\
\text { 6. APOE genotype for NCODE }\end{array}$ & $\begin{array}{l}\text { 1. Memória Lógia Imediata e Tardia } \\
\text { (WMS) } \\
\text { 2. Ascending Digit Span forward and } \\
\text { backward } \\
\text { 3. TMT A and B }\end{array}$ & $\begin{array}{l}\mathrm{SIM} \\
\mathrm{GC}>\mathrm{GD}\end{array}$ \\
\hline
\end{tabular}




\begin{tabular}{|c|c|c|c|c|}
\hline $\begin{array}{l}\text { O'Brien et } \\
\text { al, } 2004\end{array}$ & GD: $n=60$ & $\begin{array}{l}\text { 1. MADRS } \\
\text { 2.CEMDE } \\
\text { 3. MMSE } \\
\text { 4.Clinical History }\end{array}$ & $\begin{array}{l}\text { 1. Forward and Backward Digit Span; } \\
\text { 2. TMT A e B; } \\
\text { 3. Cambridge automated neuropsycho- } \\
\text { logical test battery of working memory; } \\
\text { Spatial memory }\end{array}$ & $\begin{array}{l}\mathrm{SIM} \\
\mathrm{GC}>\mathrm{GD}\end{array}$ \\
\hline $\begin{array}{l}\text { Von Gunten } \\
\text { et al., } 2005\end{array}$ & $\begin{array}{l}\text { GDD: } \\
\mathrm{n}=9 \\
\text { GD: } \\
\mathrm{n}=32\end{array}$ & $\begin{array}{l}\text { 1. MMSE } \\
\text { 2. CT or MRI brain scan; } \\
\text { 3. Ham-D } \\
\text { 4. BEHAVE. } \\
\text { 5. Interpretation of a written } \\
\text { and a drawn situation } \\
\text { 6. CDR }\end{array}$ & $\begin{array}{l}\text { 1. Immediate prose free recall } \\
\text { 2. Current intelligence score }\end{array}$ & $\begin{array}{l}\mathrm{NAO} \\
\mathrm{GD}=\mathrm{GDD}\end{array}$ \\
\hline $\begin{array}{l}\text { Sair et } \\
\text { al.,2006 }\end{array}$ & GD: $n=129$ & $\begin{array}{l}\text { 1.National Institute of Mental } \\
\text { Health Diagnostic Interview } \\
\text { Schedule } \\
\text { 2. MADRS } \\
\text { 3.MMSE }\end{array}$ & $\begin{array}{l}\text { 1. Ascending Digits Task } \\
\text { 2. forward and Backward Digit Span }\end{array}$ & $\begin{array}{l}\mathrm{SIM} \\
\mathrm{GC}>\mathrm{GD}\end{array}$ \\
\hline $\begin{array}{l}\text { Birch et al, } \\
2007\end{array}$ & GD: $n=17$ & $\begin{array}{l}\text { 1. GDS } \\
\text { 2. MMSE }\end{array}$ & $\begin{array}{l}\text { 1. WTAR (letter-number sequencing; } \\
\text { Spatial span) }\end{array}$ & $\begin{array}{l}\text { SIM } \\
\text { GC }>\text { GD Limítrofe }\end{array}$ \\
\hline $\begin{array}{l}\text { Delaloye et } \\
\text { al, } 2008\end{array}$ & GDIT: $\mathrm{n}=14$ & $\begin{array}{l}\text { 1. DRS } \\
\text { 2.GDS }\end{array}$ & $\begin{array}{l}\text { 1. French version of the reading span test } \\
\text { 2. Corsi Block Tapping Test } \\
\text { 3. TMT A and B } \\
\text { 4. Letter-Number Fluency Test }\end{array}$ & $\begin{array}{l}\text { SIM } \\
\text { GD > GDIT } \\
-\end{array}$ \\
\hline $\begin{array}{l}\text { Fischer et al, } \\
2008\end{array}$ & GD: $n=17$ & $\begin{array}{l}\text { 1.Behavioural Neurology As- } \\
\text { sessesment } \\
\text { 2. CIRS } \\
\text { 3. MMSE } \\
\text { 4.GDS }\end{array}$ & $\begin{array}{l}\text { 1. WAIS-R Digit Span; } \\
\text { 2. WMS III Spatial Span; } \\
\text { 3. TMT A and B }\end{array}$ & $\begin{array}{l}\mathrm{NAO} \\
\mathrm{GD}=\mathrm{GC}\end{array}$ \\
\hline $\begin{array}{l}\text { Lonie et al, } \\
2009\end{array}$ & GD: $\mathrm{n}=17$ & - & $\begin{array}{l}\text { 1. Dual Task (digit span e viuospacial): } \\
\text { 2. WAIS III- digits span } \\
\text { 3. Visuospacial tracking test } \\
\text { 4. TMT A and B } \\
\text { 5. HVLTR }\end{array}$ & $\begin{array}{l}\mathrm{NAO} \\
\mathrm{GC}=\mathrm{GD} \\
\mathrm{GDA}<\mathrm{GD} \\
\mathrm{GTLC}<\mathrm{GD}\end{array}$ \\
\hline $\begin{array}{l}\text { Delaloye, } \\
2010\end{array}$ & $\begin{array}{l}\text { GDR: } n=41 \\
\text { GDP: } n=30 \\
\text { GDIT: } n=11\end{array}$ & $\begin{array}{l}\text { 1. GDS } \\
\text { 2. Clinical History } \\
\text { 3. Charlson Comorbidity Index }\end{array}$ & $\begin{array}{l}\text { 1. NLS } \\
\text { 2. Reading span }\end{array}$ & $\begin{array}{l}\mathrm{NAO} \\
\mathrm{GDR}=\mathrm{GDP}= \\
\text { GDIT }\end{array}$ \\
\hline $\begin{array}{l}\text { Chuí et al, } \\
2011\end{array}$ & $\begin{array}{l}\text { GDITR: } \mathrm{n}=26 \\
\text { GDIT: } \mathrm{n}=28\end{array}$ & $\begin{array}{l}\text { 1. SCID-I, } \\
\text { 2. CDR } \\
\text { 3. HAM-D, } \\
\text { 4. Lawton- IADLS 5. CIRS }\end{array}$ & $\begin{array}{l}\text { 1. Forward and } \\
\text { backward digit spans (CDRS) } \\
\text { 2. FEP test }\end{array}$ & $\begin{array}{l}\text { SIM } \\
\text { GDIT= GDR }\end{array}$ \\
\hline
\end{tabular}

Nota . GC = Grupo Controle; GD = Grupo de deprimidos; GDR = Grupo de Deprimidos em Remissão; GDITR = Grupo de Deprimidos de ÍnicioTardio em Remissão; GDIT = Grupo de Deprimidos de Início Tardio; GDP = Grupo de Deprimidos Precoces; GDA = Grupo com Doença de Alzheimer; GDD = Grupo de Deprimidos Demenciados; GTCL = Grupo com Transtorno Cognitivo Leve; CVLT = California Verbal Learning Test; RVLT = Rey Verbal Learning Test; $\mathrm{PHQ}=$ Physical Health Questionnaire; $\mathrm{ABCD}=$ Arizona Battery for Communcation disorders of Dementia; MMSE = Mini-Mental State Exam; HAM $-\mathrm{D}=$ Hamilton Depression Scale; DRS = Demential Rating Scale; GDS = Geriatric Depression Scale; SCID = Structured Clinical Interview DSM-IVR; MADRS $=$ Montgomery Asberg Depression Rating Scale; CEMDE = Cambridge Examination for Mental Disorders of the Elderly; FEP = Finger- edge- palm test; Lawton- Instrumental Activity Daily Living Scale; CIRS = Cumulative Illness Rating Scale; CDR = Clinical Demential Rating; CGIS = Clinic Global Impression Scale; CCI = Comprehenssive Clinical Interview 
litando o seu acesso. Justifica-se essa última afirmação pela significativa correlação positiva entre o efeito de recência e o span de dígitos inverso. Os autores evidenciam que os idosos com depressão, diferentemente dos idosos com DA, ainda apresentam ativa a função da alça fonológica, porém com maior lentidão e, portanto, com menos itens do que os idosos saudáveis. Já os idosos com DA têm essa capacidade suprimida, e apenas conseguem utilizar as informações muito recentemente apresentadas, uma vez que o ensaio articulatório da alça fonológica falha na transferência para a memória de longo prazo (Foldi et al., 2003).

Assim, pode-se pensar que as diferenças no funcionamento da MT de idosos deprimidos e idosos com DA, ou outra demência, são um tanto específicas e serão influenciadas pela tarefa utilizada na investigação. É fato, porém, que os autores encontram maiores prejuízos na MT de idosos com DA e outras demências do que em idosos apenas deprimidos. Artigos sinalizam prejuízos significativos dos idosos com DA no armazenamento da informação verbal e episódica (Foldi et al., 2003; Lonie et al., 2009) na comparação com idosos deprimidos.

Existem alguns fatores intervenientes nos resultados encontrados. Um deles seria o fato dos artigos serem muito superficiais ao definir o conceito de MT e a maior parte não se preocupa em definir qual modelo estão utilizando. Ademais, a MT, segundo o constructo de Baddeley (2009), possui quatro componentes principais, cada um lidando com informações específicas e com funções individuais. Isso nos faz perceber que os estudos investigados não se preocuparam em esclarecer tais diferenças, ou em esclarecer a que componente da MT estão se referindo ao optar por determinado teste. Assim, se considerarmos o conceito multidimensional da MT proposto por Baddeley (2000), a maior parte dos estudos não avaliaram a MT de forma apropriada, ou seja, considerando toda sua multidimensionalidade.

No referente às avaliações neuropsicológicas dos artigos incluídos nessa revisão, as tarefas Span de Dígitos direto e, especialmente, o Dígitos inverso, além do Trail Making Test (TMT) A e B são os testes com maior incidência nos estudos, respectivamente: nove e oito ocorrências. O primeiro teste apresenta estímulos auditivos e verbais, sendo sua primeira parte relacionada à alça fonológica e a segunda ao executivo central. Já o TMT, cujos estímulos são visuais, em sua forma $\mathrm{A}$, apenas mensura a atenção, enquanto em sua forma $\mathrm{B}$, avalia aspectos executivos, podendo ser considerado um teste para avaliar o executivo central. Portanto, a utilização de apenas um desses testes configura-se em uma forma bastante parcial, e sintetizada, de avaliação da MT, considerando seu modelo multidimensional (Baddeley, 2000).

Excepcionalmente, o estudo de Sair et al. (2006), que apresenta o Ascending Digit Test (ADT) é muito claro ao mencionar que este teste avalia especificamente o executivo central da MT. Em seu estudo, os autores fazem uma comparação do desempenho de idosos deprimidos e saudáveis no teste ADT. Eles concluem que efetivamente idosos deprimidos tiveram pior desempenho no teste ADT e se referem ao componente executivo central do modelo de MT de Baddeley como o maior prejudicado pelos efeitos da depressão em idosos (Sair et al., 2006). Além desses autores,
Elderkin-Thompson et al. (2003) também concluíram que o executivo central seria o componente afetado em idosos deprimidos. Os estudos de Nebes et al. $(2001,2003)$ investigam o desempenho de idosos deprimidos em duplas-tarefas (duas tarefas cognitivas distintas realizadas simultaneamente). Eles evidenciam que os idosos tiveram seu desempenho prejudicado em relação a tarefas únicas quando foram incentivados a realizar duplas-tarefas. Esses autores observaram prejuízo maior na tarefa visuomotora em prol da manutenção do desempenho na tarefa verbal. Eles atribuem esse efeito como consequente de uma dificuldade de coordenação e seleção do executivo central (Nebes et al., 2001).

No que se refere à idade de início da depressão, cinco estudos esclarecem o tipo de depressão que estão estudando (Chui, Cheung, \& Lam, 2011; Delaloye et al., 2008; Delaloye et al., 2009; Murray, 2002; Simpson et al., 2001). Murray (2002) menciona que investigou idosos com depressão maior recorrente, já Delaloye et al. (2008, 2009) e Chui et al. (2011) mencionam que estão preocupados em estudar idosos com depressão de início após os 60 anos. Um dos estudos de Delaloye et al. (2008) sugere que nos idosos com depressão de início tardio, déficits na MT impactam a capacidade da memória episódica, inferindo que os déficits nesta última poderiam ser consequentes aos prejuízos da MT. O mesmo já havia sido mencionado por Nebes et al. em 2000, porém este último associa a capacidade de velocidade do processamento e a MT como responsáveis pelas dificuldades observadas na memória episódica, enquanto Delaloye et al. (2009) mencionam apenas a MT como elemento fundamental para memória episódica.

Também Birch e Davidson (2007), ao estudarem a memória autobiográfica de idosos deprimidos, demonstraram que a MT é de especial importância para a recuperação dessa memória, especialmente seu componente visuoespacial. Dificuldades nessa função podem impactar significativamente a capacidade de recuperação de memórias autobiográficas.

\section{Considerações Finais}

Evidencia-se nesta revisão que alterações na MT são associadas à depressão em idosos. Além disso, destaca-se também que estas, tendem a permanecerem após a remissão dos sintomas (Delaloye et al., 2010; Nebes et al., 2003). Investigações sustentam que essas perdas seriam decorrentes de alterações neuroestruturais relacionadas à depressão (Simpson et al., 2001). Entretanto, essas variam de acordo com a maneira como a depressão se apresenta. Depressão de início tardio vem sendo associada a alterações orgânicas gerais que interferem em alterações no volume encefálico frontal e parietal (Simpson et al., 2001). Já as depressões recorrentes, que se repetem durante o envelhecimento, podem ser mais associadas a alterações nas estruturas do hipocampo (O’Brien et al., 2004). Entretanto, isso ainda é controverso, sendo necessários mais estudos que esclareçam esse dado.

É fato que a maior parte dos estudos incluídos nesta revisão não se preocupou em definir o período de início da depressão, o que denota uma necessidade de maior rigor por parte dos pesquisadores nesse sentido em futuros es- 
tudos. Ademais, os artigos são pouco específicos quanto à sua definição de MT e ainda mais quanto à escolha do teste utilizado para avaliar essa função, muitas feitas por apenas um instrumento com o objetivo de auferir a MT. Observa-se, portanto, o uso de poucos instrumentos e geralmente atividades que apenas remetem a um ou dois componentes da MT, mas não ao modelo multidimensional como conceituado por Baddeley (2000). Assim, ao se referir a este constructo, os autores devem ser mais cuidadosos, esclarecendo de forma específica a qual componente estão se referindo. Assim poder-se-ia compreender melhor a relação entre a depressão no idoso e a MT.

Enfim, a importância de investigações que se aprofundem nessa temática é evidenciada, uma vez que o impacto das alterações da MT na vida do indivíduo deprimido pode provocar dependência funcional e uma perda significativa da qualidade de vida, sendo ainda fator de risco altamente relacionado à consequente apresentação de quadros demenciais, levando inclusive alguns autores a hipotetizar que as perdas cognitivas de idosos deprimidos seriam na realidade primeiras manifestações degenerativas orgânicas (Steffens et al., 2006; von Gunten et al., 2005).

\section{Referências}

Baddeley, A. (2000). The episodic buffer: a new component of working memory? Trends in Cognitive Sciences, 4(11), 417423. doi: S1364-6613(00)01538-2 [pii]

Baddeley, A. (2009). Working memory. In A. Baddeley, M. Anderson \& M. Eysenck (Eds.), Memory (pp. 41-68). Hove: Psychology Press.

Birch, L. S., \& Davidson, K. M. (2007). Specificity of autobiographical memory in depressed older adults and its relationship with working memory and IQ. The British Journal of Clinical Psychology, 46(Pt 2), 175-186. doi: 10.1348/014466506X119944

Chui, W. W., Cheung, E. F., \& Lam, L. C. (2011). Neuropsychological profiles and short-term outcome in late-onset depression. International Journal of Geriatric Psychiatry, 26(5), 458-465. doi: $10.1002 /$ gps. 2548

Delaloye, C., Baudois, S., de Bilbao, F., Dubois Remund, C., Hofer, F., Lamon, M., . . . Giannakopoulos, P. (2008). Cognitive impairment in late-onset depression. Limited to a decrement in information processing resources? European Neurology, 60(3), 149-154. doi: 000144086 [pii] 10.1159/000144086

Delaloye, C., de Bilbao, F., Moy, G., Baudois, S., Weber, K., Campos, L., \& Gold, G. (2009). Neuroanatomical and neuropsychological features of euthymic patients with bipolar disorder. American Journal of Geriatric Psychiatry, 17(12), 1012-1021. doi: 00019442-200912000-00004 [pii] 10.1097/ JGP.0b013e3181b7f0e2

Delaloye, C., Moy, G., de Bilbao, F., Baudois, S., Weber, K., Hofer, F., \& Gold, G. (2010). Neuroanatomical and neuropsychological features of elderly euthymic depressed patients with early-and-late-onset. Journal of the Neurological Sciences, 299(1-2), 19-23. doi: S0022-510X(10)00413-2 [pii] 10.1016/j.jns.2010.08.046
Elderkin-Thompson, V., Kumar, A., Bilker, W. B., Dunkin, J. J., Mintz, J., Moberg, P. J., ... Gur, R. E. (2003). Neuropsychological deficits among patients with late-onset minor and major depression. Archives of Clinical Neuropsychology, 18(5), 529549. doi: S0887617703000222 [pii]

Fischer, C., Schweizer, T. A., Atkins, J. H., Bozanovic, R., Norris, M., Herrmann, N., ... Rourke, S. B. (2008). Neurocognitive profiles in older adults with and without major depression. International Journal of Geriatric Psychiatry, 23(8), 851-856. doi: 10.1002/gps.1994

Fiske, A., Wetherell, J. L., \& Gatz, M. (2009). Depression in older adults. Annual Review of Clinical Psychology, 5, 363-389. doi: 10.1146/annurev.clinpsy.032408.153621

Foldi, N. S., Brickman, A. M., Schaefer, L. A., \& Knutelska, M. E. (2003). Distinct serial position profiles and neuropsychological measures differentiate late life depression from normal aging and Alzheimer's disease. Psychiatry Research, 120(1), 71-84. doi: S016517810300163X [pii]

Goffaux, P., Phillips, N. A., Sinai, M., \& Pushkar, D. (2008). Neurophysiological measures of task-set switching: effects of working memory and aging. The Journals of Gerontology: Series B, Psychology Social Sciences, 63(2), P57-66. doi: 63/2/P57 [pii]

Herrmann, L. L., Goodwin, G. M., \& Ebmeier, K. P. (2007). The cognitive neuropsychology of depression in the elderly. Psychological Medicine, 37(12), 1693-1702. doi: S0033291707001134 [pii] 10.1017/S0033291707001134

Lonie, J. A., Tierney, K. M., Herrmann, L. L., Donaghey, C., O'Carroll, R. E., Lee, A., \& Ebmeier, K. P. (2009). Dual task performance in early Alzheimer's disease, amnestic mild cognitive impairment and depression. Psychological Medicine, 39(1), 23-31. doi: S0033291708003346 [pii] 10.1017/ S0033291708003346

Luo, L., \& Craik, F. I. (2008). Aging and memory: A cognitive approach. Canadian Journal of Psychiatry, 53(6), 346-353.

Mondal, S., Sharma, V. K., Das, S., Goswami, U., \& Gandhi, A. (2007). Neuro-cognitive functions in patients of major depression. Indian Journal of Physiology Pharmacology, $51(1), 69-75$.

Murray, L. (2002). Cognitive distinctions between depression and early Alzheimer's disease in the elderly. Aphasiology, 16(4/5/6), 573-585. doi: 10.1080/02687030244000293

Nebes, R. D., Butters, M. A., Houck, P. R., Zmuda, M. D., Aizenstein, H., Pollock, B. G., Reynolds, C. F. (2001). Dual-task performance in depressed geriatric patients. Psychiatry Research, 102(2), 139-151. doi: S01651781(01)00244-X [pii]

Nebes, R. D., Butters, M. A., Mulsant, B. H., Pollock, B. G., Zmuda, M. D., Houck, P. R., \& Reynolds, C. F. (2000). Decreased working memory and processing speed mediate cognitive impairment in geriatric depression. Psychological Medicine, 30(3), 679-691.

Nebes, R. D., Pollock, B. G., Houck, P. R., Butters, M. A., Mulsant, B. H., Zmuda, M. D., \& Reynolds, C. F. (2003). Persistence of cognitive impairment in geriatric patients following antidepressant treatment: A randomized, doubleblind clinical trial with nortriptyline and paroxetine. Jounal of Psychiatry Research, 37(2), 99-108. doi: S0022395602000857 [pii] 
Nyberg, L., Dahlin, E., Stigsdotter Neely, A., \& Bäckman, L. (2009). Neural correlates of variable working memory load across adult age and skill: Dissociative patterns within the fronto-parietal network. Scandinavian Journal of Psychology, 50(1), 41-46. doi: SJOP678 [pii] 10.1111/j.1467-9450.2008.00678.x

O’Brien, J. T., Lloyd, A., McKeith, I., Gholkar, A., \& Ferrier, N. (2004). A longitudinal study of hippocampal volume, cortisol levels, and cognition in older depressed subjects. American Journal of Psychiatry, 161(11), 2081-2090. doi: 161/11/2081 [pii] 10.1176/appi.ajp.161.11.2081

Owen, A. M., McMillan, K. M., Laird, A. R., \& Bullmore, E. (2005). N-back working memory paradigm: A meta-analysis of normative functional neuroimaging studies. Human Brain Mapping, 25(1), 46-59. doi: 10.1002/hbm.20131

Rozenthal, M., Laks, J., \& Egekhardt, E. (2004). Aspectos neuropsicológicos da depressão. Revista de Psiquiatria do Rio Grande do Sul, 26(2), 204-212.

Sair, H. I., Welsh-Bohmer, K. A., Wagner, H. R., \& Steffens, D. C. (2006). Ascending digits task as a measure of executive function in geriatric depression. Journal of Neuropsychiatry Clinical Neurosciences, 18(1), 117-120. doi: 18/1/117 [pii] 10.1176/appi.neuropsych.18.1.117

Simpson, S. W., Baldwin, R. C., Burns, A., \& Jackson, A. (2001). Regional cerebral volume measurements in late-life depression: relationship to clinical correlates, neuropsychological impairment and response to treatment. International Journal of Geriatric Psychiatry, 16(5), 469-476.
Steffens, D. C., Otey, E., Alexopoulos, G. S., Butters, M. A., Cuthbert, B., Ganguli, M., .. Y Yesavage, J. (2006). Perspectives on depression, mild cognitive impairment, and cognitive decline. Archives of General Psychiatry, 63(2), 130-138. doi: 63/2/130 [pii] 10.1001/archpsyc.63.2.130

Steffens, D. C., Welsh-Bohmer, K. A., Burke, J. R., Plassman, B. L., Beyer, J. L., Gersing, K. R., \& Potter, G. G. (2004). Methodology and preliminary results from the neurocognitive outcomes of depression in the elderly study. Journal of Geriatric Psychiatry Neurology, 17(4), 202-211. doi: 17/4/202 [pii] 10.1177/0891988704269819

Von Gunten, A., Giannakopoulos, P., \& Duc, R. (2005). Cognitive and demographic determinants of dementia in depressed patients with subjective memory complaints. European Neurology, 54(3), 154-158. doi: 90104 [pii] 10.1159/000090104

Recebido em 05.11.2011

Primeira decisão editorial em 17.07.2012

Versão final em 24.08.2011

Aceito em 19.11.2011 